\section{Hydroxyurea to lower transcranial Doppler velocities and prevent primary stroke: the Uganda NOHARM sickle cell anemia cohort}

In sub-Saharan Africa, sickle cell anemia (SCA) remains a significant public health problem with high mortality: an estimated $50-90 \%$ of affected children die before 5 years of age. ${ }^{1}$ A high prevalence of stroke is particularly devastating for children and has substantial morbidity. ${ }^{2,3}$ Hydroxyurea is now recommended across the lifespan as a safe and effective disease-modifying therapy for SCA, 4,5 but is not routinely available in sub-Saharan Africa. ${ }^{6}$

NOHARM (NCT01976416), a randomized doubleblinded, placebo-controlled trial, was among the first prospective studies to investigate hydroxyurea treatment for children with SCA living in a malaria endemic region within Africa. The NOHARM trial consisted of a blinded phase (Year 1) and an open-label phase (Year 2). Initially children 1.00-4.99 years of age were randomized either to placebo or fixed-dose hydroxyurea $(20 \mathrm{mg} / \mathrm{kg} / \mathrm{day})$. The Year 1 results demonstrated that short-term hydroxyurea treatment was both safe and efficacious in this young patient population living in a malarial endemic region. [8] We now analyze Year 2 data from the openlabel phase, in which all NOHARM participants received hydroxyurea at $20 \mathrm{mg} / \mathrm{kg} / \mathrm{day}$, to compare the effects of hydroxyurea on SCA-related morbidity and also to provide serial transcranial Doppler (TCD) data, which were not included in the original randomized report.

NOHARM was conducted at the Mulago Hospital Sickle Cell Clinic in Kampala, Uganda. A detailed description of the study design, site, study participants, and process for the blinded phase has previously been published. ${ }^{7,8}$ All study participants were given the opportunity to receive open-label hydroxyurea for 12 months, after the first year of randomized treatment, since there was no evidence from interim analysis of harm for the hydroxyurea arm compared to placebo. Study outcomes included rates of clinical malaria, clinical sickle cell-related adverse events (AE), serious adverse events, hematological toxicities, and laboratory benefits, as well as TCD velocities. Ethical and regulatory approvals were obtained from appropriate institutional review and regulatory boards, and informed consent was obtained from the parents or legal guardians..$^{, 10}$

Study participants received two TCD examinations, one at the end of randomized treatment (month 10 or 12) and again at the completion of open-label treatment (month 22 or 24). TCD screening was not performed at baseline because TCD equipment, training, and examiner certification were not available at study enrollment. All TCD examinations were performed by one certified examiner and the results were read offsite by experts as described. ${ }^{9}$ The maximum time-averaged mean velocity (TAMV) was recorded in the middle cerebral or internal carotid arteries, and classified as normal $(<170 \mathrm{~cm} / \mathrm{sec})$, conditional $(170-199 \mathrm{~cm} / \mathrm{sec})$ or abnormal $(\geq 200 \mathrm{~cm} / \mathrm{sec})$, with velocities in the conditional or abnormal ranges indicating greater risk of stroke. ${ }^{10}$ Children with abnormal readings had a confirmatory repeat TCD exam performed within two months; if that abnormal exam was confirmed, they received monthly transfusions for primary stroke prevention.

Continuous measures were described as mean (standard deviation [SD]), and treatment arms were compared using $t$-tests, or a paired $t$-test for the same individuals. Categorical measures were described using percent or frequency, and compared using the Pearson $\chi^{2}$ test with Yates correction or Fisher's exact test. Rates of adverse events including malaria between arms were presented and compared by the incidence rate ratio (IRR) with $95 \%$ confidence intervals (CI), which were calculated using Poisson regression. Study data were analyzed using $\mathrm{R}$ (Vienna, Austria, version 3.2.4). Statistical significance was assessed at the 0.05 level.

All but one family of the 199 children who completed the blinded randomized phase of NOHARM enrolled into the open-label phase (average age $3.3 \pm 0.9$ years), and 192 of $198(97.0 \%)$ completed the open-label treatment phase (Figure 1). At the end of blinded study treatment,185 TCD examinations were completed, of which 164 were suitable for analysis ( 81 hydroxyurea, 83 placebo). Overall $80 \%$ of children on hydroxyurea had normal TCD velocities with a median TAMV of $138 \mathrm{~cm} / \mathrm{sec}$ compared to $77 \%$ normal exams for children on placebo

Table 1. Laboratory parameters during open label hydroxyurea treatment. Analysis includes only laboratory values with paired samples at months 12 and 24 that span the open-label treatment period.

\begin{tabular}{|c|c|c|c|c|c|c|}
\hline \multirow[b]{2}{*}{ Year 1 treatment before open label } & \multicolumn{2}{|c|}{ Month 24 Lab Values } & \multicolumn{4}{|c|}{ Change from Month 12} \\
\hline & $\begin{array}{l}\text { Hydroxyurea } \\
\text { Year } 1\end{array}$ & $\begin{array}{l}\text { Placebo } \\
\text { Year } 1\end{array}$ & $\mathbf{P}$ & $\begin{array}{l}\text { Hydroxyurea } \\
\text { Year } 1\end{array}$ & $\begin{array}{l}\text { Placebo } \\
\text { Year } 1\end{array}$ & $P$ \\
\hline Hemoglobin (g/dL) & $8.7 \pm 1.7$ & $8.8 \pm 1.1$ & 0.4149 & $0.0 \pm 1.7$ & $1.4 \pm 1.0$ & $<0.0001$ \\
\hline Mean corpuscular volume (MCV, fL) & $91 \pm 10$ & $92 \pm 9$ & 0.3771 & $3 \pm 7$ & $12 \pm 6$ & $<0.0001$ \\
\hline Fetal hemoglobin $[(\mathrm{HbF}) /(\mathrm{F}+\mathrm{S}), \%)]$ & $25.1 \pm 9.3$ & $26.8 \pm 8.8$ & 0.1807 & $2.3 \pm 6.6$ & $16.4 \pm 7.1$ & $<0.0001$ \\
\hline Enrollment age below median & $26.2 \pm 8.4$ & $27.4 \pm 9.2$ & 0.5014 & $2.4 \pm 7.2$ & $15.3 \pm 7.1$ & $<0.0001$ \\
\hline Enrollment age above median & $23.6 \pm 10.3$ & $26.3 \pm 8.5$ & 0.1820 & $2.1 \pm 5.7$ & $17.4 \pm 7.0$ & $<0.000$ \\
\hline Absolute reticulocyte count (ARC, x10\%/L) & $221 \pm 93$ & $214 \pm 82$ & 0.6048 & $-28 \pm 107$ & $-173 \pm 129$ & $<0.0001$ \\
\hline White blood cell count (WBC, x109\%/) & $11.4 \pm 4.2$ & $11.2 \pm 4.1$ & 0.7002 & $-2.3 \pm 5.0$ & $-6.8 \pm 5.0$ & $<0.0001$ \\
\hline Absolute neutrophil count (ANC, x109/L) & $4.5 \pm 2.1$ & $4.4 \pm 2.0$ & 0.7171 & $-0.7 \pm 2.7$ & $-2.2 \pm 2.9$ & 0.0002 \\
\hline Platelets $($ x 109) & $370 \pm 180$ & $382 \pm 175$ & 0.6475 & $0 \pm 174$ & $-61 \pm 178$ & 0.0164 \\
\hline Alanine transferase (ALT, U/L) & $19 \pm 14$ & $20 \pm 12$ & 0.7961 & $-1 \pm 15$ & $2 \pm 12$ & 0.2525 \\
\hline Creatinine $(\mathrm{mg} / \mathrm{dL})$ & $0.43 \pm 0.13$ & $0.39 \pm 0.15$ & 0.0217 & $0.11 \pm 0.15$ & $0.07 \pm 0.16$ & 0.1114 \\
\hline
\end{tabular}

There were 97 for children who received hydroxyurea in Year 1, and 95 who received placebo in Year 1, who had paired samples during the Year 2 open label hydroxyurea treatment period. 
and median TAMV of $150 \mathrm{~cm} / \mathrm{sec},(P=0.05$ for TAMV comparison). At the end of the open-label phase, 187 TCD exams had been conducted and all were suitable for analysis. At that point $89 \%$ had normal velocities, with a median TAMV of $137 \mathrm{~cm} / \mathrm{sec}$ in both original treatment cohorts. Only one child in NOHARM suffered a clinical stroke, 17 days after completing placebo and transitioning to open-label hydroxyurea treatment.

In addition to lower TCD velocities, hydroxyurea treatment also led to expected hematological benefits, which were rapidly achieved in children previously on placebo, and maintained in children previously on hydroxyurea (Table 1). Among 99 children previously on placebo, the overall AE, sickle cell-related AE, and hospitalizations were significantly lower after open-label hydroxyurea (Table 2). For 99 children previously on hydroxyurea, low clinical AE rates were maintained. Malaria rates were low and did not differ between the two groups (Table 2). Three deaths (one acute chest syndrome, one presumed sepsis, one acute splenic sequestration) occurred in children originally assigned to placebo and two deaths (one acute chest syndrome, one sepsis) occurred in children originally assigned to hydroxyurea. This compared to one death in the placebo arm and two deaths in the hydroxyurea arm in the randomized phase (Figure 1).

Laboratory AE and dose-limiting toxicities (DLT) were infrequent during the year of the open-label hydroxyurea treatment (Table 2). The majority of DLT occurred during the course of a clinical AE. Two children originally randomized to hydroxyurea treatment developed chronic marked splenomegaly $(5-8 \mathrm{~cm}$ below the left costal margin) that persisted during Year 2, and together accounted for six DLT based on episodes of anemia, reticulocytopenia and thrombocytopenia. At each event, hydroxyurea treatment was temporarily held, and both children eventually underwent successful elective surgical splenectomy. There were no episodes of renal or hepatic toxicity noted.

The present study adds to previously published results of hydroxyurea benefits for children with SCA living in sub-Saharan Africa, ${ }^{10,11}$ demonstrating significantly lower average TCD velocities on hydroxyurea compared to placebo, and only one stroke occurring in a child who received placebo in the randomized phase of the trial. During both treatment phases of the NOHARM trial, we observed lower TCD velocities for children receiving hydroxyurea treatment, along with a very low incidence of primary stroke. This observation is especially important for Uganda and other parts of sub-Saharan Africa, where stroke is common and has devastating clinical and economic implications. , $^{2,12,13}$ Hydroxyurea treatment at maximal tolerated dose (MTD) was previously shown to lower TCD velocities in the United States, ${ }^{14}$ but our NOHARM data are the first to document a similar benefit from fixed dose $(20 \mathrm{mg} / \mathrm{kg} /$ day) hydroxyurea in Africa.

Table 2. Clinical adverse events and laboratory toxicities in the NOHARM trial, based on original blinded treatment assignment, with incidence rate ratio (IRR) and $95 \%$ confidence intervals.

\begin{tabular}{|c|c|c|c|c|c|c|c|}
\hline & $\begin{array}{r}\text { Children Rand } \\
\text { Open }\end{array}$ & $\begin{array}{l}\text { omized to Hydr } \\
\text {-Label Hydroxy }\end{array}$ & $\begin{array}{l}\text { droxyurea } \rightarrow \\
\text { gyurea }\end{array}$ & $\begin{array}{r}\text { Childrer } \\
\text { Ope }\end{array}$ & $\begin{array}{l}\text { n Randomized t } \\
\text { n-Label Hydroxy }\end{array}$ & $\begin{array}{l}\text { to Placebo } \rightarrow \\
\text { yurea }\end{array}$ & All Children \\
\hline & $\begin{array}{l}\text { Y1 Events } \\
\text { (Participants) }\end{array}$ & $\begin{array}{l}\text { Y2 Events } \\
\text { (Participants) }\end{array}$ & $\begin{array}{l}\text { Y2 Events: } \\
\text { Y1 Events } \\
\text { IRR }(95 \% \text { CI) }\end{array}$ & $\begin{array}{l}\text { Y1 Events } \\
\text { (Participants) }\end{array}$ & $\begin{array}{l}\text { Y2 Events } \\
\text { (Participants) }\end{array}$ & $\begin{array}{l}\text { Y2 Hydroxyurea: } \\
\text { Y1 Placebo } \\
\text { IRR ( } 95 \% \text { CI) }\end{array}$ & $\begin{array}{l}\text { Y2 Events HU HU: } \\
\text { Y2 Events PL HU } \\
\text { IRR ( } 95 \% \text { CI) }\end{array}$ \\
\hline Clinical Adverse Events & $232(76)$ & $185(74)$ & $0.81(0.62-1.05)$ & $308(88)$ & $156(61)$ & $0.51(0.39-0.67)^{*}$ & $1.18(0.87-1.58)$ \\
\hline Rate per Patient-Year & 2.3 & 1.8 & $\mathrm{NA}$ & 3.0 & 1.6 & NA & NA \\
\hline Serious Adverse Events & $6(6)$ & $8(5)$ & $1.49(0.48-4.62)$ & $6(6)$ & $4(4)$ & $0.74(0.25-2.19)$ & $1.98(0.47-8.44)$ \\
\hline Malaria & $5(3)$ & $6(4)$ & $1.22(0.22-6.84)$ & $7(7)$ & $6(6)$ & $0.86(0.29-2.57)$ & $0.99(0.26-3.86)$ \\
\hline Clinical Sepsis \# & $8(6)$ & $5(5)$ & $0.64(0.19-2.15)$ & $16(13)$ & $4(4)$ & $0.25(0.08-0.79)^{* *}$ & $1.24(0.34-4.48)$ \\
\hline Deaths & $2(2)$ & $2(2)$ & $1.00(0.14-6.96)$ & $1(1)$ & $3(3)$ & $3.03(0.32-28.8)$ & $0.66(0.11-3.93)$ \\
\hline Sickle-related adverse events & $82(45)$ & $65(49)$ & $0.80(0.58-1.11)$ & $138(65)$ & $59(38)$ & $0.43(0.31-0.60)^{*}$ & $1.09(0.74-1.62)$ \\
\hline Vaso-occlusive crisis (dactylitis) & $58(38)$ & $51(41)$ & $0.89(0.64-1.24)$ & $106(59)$ & $45(32)$ & $0.43(0.30-0.62)^{*}$ & $1.12(0.74-1.72)$ \\
\hline Acute chest syndrome/pneumonia & $24(21)$ & $13(12)$ & $0.55(0.27-1.11)$ & $32(24)$ & $13(13)$ & $0.41(0.21-0.80)^{* *}$ & $0.99(0.47-2.10)$ \\
\hline Acute Splenic Sequestration & $0(0)$ & $1(1)$ & NA & $0(0)$ & $0(0)$ & NA & NA \\
\hline Stroke & $0(0)$ & $0(0)$ & NA & $0(0)$ & $1(1)$ & NA & NA \\
\hline Grade 3 or above & $10(9)$ & $8(8)$ & $0.81(0.34-1.93)$ & $16(14)$ & $12(9)$ & $0.76(0.34-1.70)$ & $0.66(0.26-1.69)$ \\
\hline Composite $^{\wedge}$ & 47 & 53 & $1.14(0.89-1.47)$ & 71 & 42 & $0.60(0.47-0.76)^{*}$ & $1.25(0.93-1.68)$ \\
\hline Transfusions & $14(12)$ & $17(12)$ & $1.19(0.68-2.09)$ & $18(17)$ & $17(6)$ & $0.95(0.34-2.70)$ & $0.99(0.31-3.21)$ \\
\hline Hospitalizations & $20(12)$ & $14(10)$ & $0.70(0.32-1.53)$ & $35(28)$ & $5(5)$ & $0.14(0.06-0.36)^{*}$ & $2.78(0.91-8.44)$ \\
\hline Dose-limiting toxicities (DLT) & $21(15)$ & $26(14)$ & $1.23(0.69-2.19)$ & $17(13)$ & $9(4)$ & $0.54(0.16-1.84)$ & $2.87(0.86-9.51)$ \\
\hline Anemia & $6(4)$ & $8(6)$ & $1.29(0.54-3.07)$ & $8(8)$ & $2(2)$ & $0.25(0.05-1.19)$ & $3.97(0.77-20.37)$ \\
\hline Reticulocytopenia & $1(1)$ & $8(7)$ & $7.91(1.28-48.7)^{* *}$ & $5(5)$ & $2(2)$ & $0.41(0.08-2.09)$ & $3.97(0.83-18.92)$ \\
\hline Neutropenia & $2(2)$ & $5(5)$ & $2.54(0.49-13.1)$ & $0(0)$ & $5(2)$ & NA & $0.99(0.16-6.09)$ \\
\hline Thrombocytopenia & $12(11)$ & $5(3)$ & $0.42(0.12-1.42)$ & $4(4)$ & $0(0)$ & NA & $\mathrm{NA}$ \\
\hline
\end{tabular}

${ }^{*}$ Clinical sepsis refers to measured fever and ill appearance, receiving IV antibiotics. ${ }^{\wedge}$ Protocol-specified composite endpoint includes vaso-occlusive pain, dactylitis, acute chest syndrome, acute splenic sequestration, or transfusions. Year 1 data were previously published. ${ }^{*}: P<0.001$ for incidence rate ratio (IRR) of a particular clinical or laboratory event; **: $P<0.05$ for IRR of particular clinical or laboratory event. NA: not analyzed. 


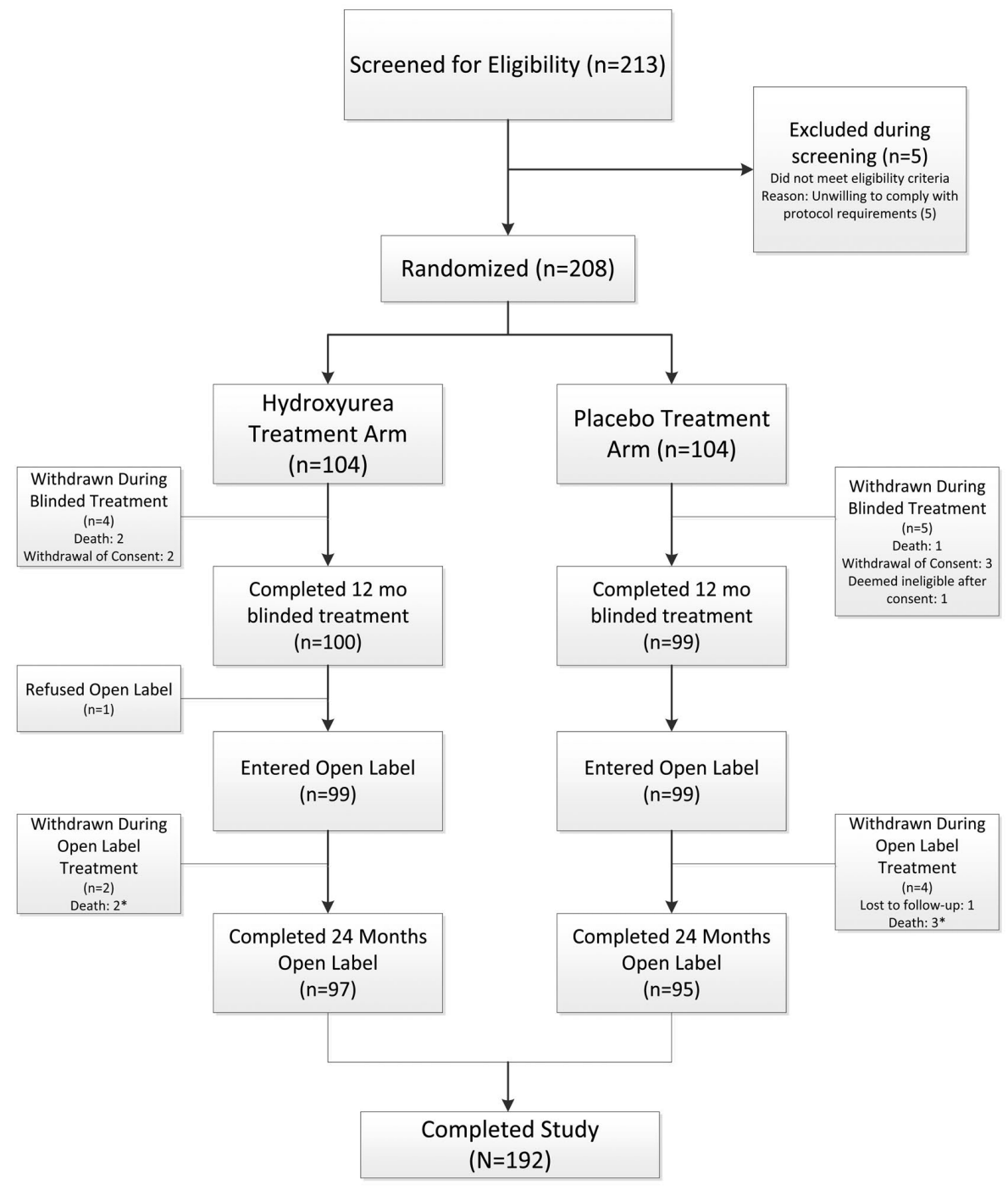

Figure 1. CONSORT diagram of the NOHARM trial. A total of 199 young children with sickle cell anemia (SCA) completed Year 1, either fixed-dose hydroxyurea or placebo treatment. All but one then entered the open-label treatment phase and 192 completed the study after Year 2 of hydroxyurea treatment. There were five deaths in the open-label treatment phase; including two $(*)$ that occurred within the scheduled 30 day window after the final end-ofstudy visit.

In addition to TCD velocity reduction and stroke protection, multiple laboratory and clinical benefits of openlabel hydroxyurea were quickly achieved in children previously randomized to placebo. Our data provide almost 200 additional patient-years of hydroxyurea treatment experience in Africa, documenting consistent and sustained laboratory and clinical benefits from open-label hydroxyurea during the second year, regardless of the original treatment assignment. For children previously randomized to hydroxyurea, continued treatment was very well-tolerated, and associated with relatively few laboratory AE or DLT throughout the extended treatment period.

A few children in NOHARM developed substantial splenomegaly (typically $5-8 \mathrm{~cm}$ below the left costal margin on physical exam) and in two cases, the resulting hypersplenism with associated anemia and thrombocytopenia led to repeated pauses in treatment due to low laboratory values. These were not likely true DLT that were attributable to excessive drug-induced marrow suppression, but still prevented consistent daily treatment. Only after elective surgical splenectomy, a complicated and high-risk procedure in lower-resource settings, were these children able to restart daily hydroxyurea without frequent laboratory toxicities. By reducing erythrocyte sickling, early initiation of hydroxyurea should decrease splenic infarction and thus could preserve splenic tissue. ${ }^{15}$ Potentially this improvement in erythrocyte shape and function contributes to hypersplenism in some children, although reduced sequestration of sickled erythrocytes should also reduce the risk of splenomegaly and hypersplenism. The malaria parasite burden in African children in malaria endemic areas may also modify splenic behavior. Prospective analysis of splenic size and function during hydroxyurea treatment is an important consideration for future studies within sub-Saharan Africa, and could help define the optimal starting age and dosing goals.

In summary, the NOHARM trial provides additional evidence for the safety and efficacy of short-term hydroxyurea treatment for SCA in sub-Saharan Africa within a clinical trial setting. Data from both the blinded treatment phase and the open-label treatment phase demonstrate clinical and hematological benefits of hydroxyurea in a malarial endemic region, including evidence of reduced TCD velocities and primary stroke prevention. Additional TCD screening programs and clinical trials are urgently needed in sub-Saharan Africa to document the feasibility and benefits of hydroxyurea treatment for children with conditional or even abnormal TCD velocities, given the challenges of providing chronic blood transfusions. To help prevent primary stroke in this high-risk population, future studies should also focus on optimal 
dosing strategies and monitoring regimens, in an effort to determine the overall feasibility and safety of introducing hydroxyurea therapy for SCA widely across sub-Saharan Africa.

Robert O. Opoka, Heather A. Hume, ${ }^{1,2}$ Teresa S. Latham, ${ }^{3}$ Adam Lane, ${ }^{3,4}$ Olatundun Williams, ${ }^{5}$ Jennifer Tymon, ${ }^{6}$ Maria Nakafeero, ${ }^{3,7}$ Phillip Kasirye, ${ }^{1}$ Christopher M. Ndugwa, Chandy C. John, ${ }^{8 \#}$ and Russell E. Ware ${ }^{3,4,9 \#}$ ${ }^{*} C C J$ and REW contributed equally as co-senior authors.

${ }^{1}$ Department of Paediatrics and Child Health, Makerere University, Kampala Uganda; ${ }^{2}$ Département de Pédiatrie, Centre Universitaire Ste-Justine, Université de Montréal, Montréal, Canada; ${ }^{3}$ Division of Hematology, Department of Pediatrics, Cincinnati Children's Hospital Medical Center, Cincinnati, OH, USA; ${ }^{4}$ University of Cincinnati College of Medicine, Cincinnati, OH, USA; ${ }^{5}$ Ann and Robert H. Lurie Children's Hospital of Chicago, Chicago, IL, USA; ${ }^{6}$ St. Luke's University Health Network, Bethlehem PA; ${ }^{7}$ Global Health Uganda, Kampala, Uganda; ${ }^{8}$ Ryan White Center for Infectious Diseases and Global Health, Department of Pediatrics, University of Indiana, Indianapolis, IN; ${ }^{9}$ Global Health Center, Cincinnati Children's Hospital Medical Center, Cincinnati, OH, USA

Correspondence: RUSSELL E. WARE - russell.ware@cchmc.org doi:10.3324/haematol.2019.231407

Acknowledgments: the authors thank all of the patients who volunteered to participate in this study as well as the Mulago Hospital Sickle Cell Clinic and Global Health Uganda staff for conducting study work. The Data Coordinating Center staff at Cincinnati Children's Hospital Medical Center set up the study database and monitored all study data.

Funding: Doris Duke Charitable Foundation provided funds for the study (ICRA 2013139) and Addmedica Inc. (Paris, France) donated hydroxyurea (Siklos@) for the NOHARM trial.

Information on authorship, contributions, and financial \& other disclosures was provided by the authors and is available with the online version of this article at WWW. haematologica.org.

\section{References}

1. Grosse SD, Odame I, Atrash HK, Amendah DD, Piel FB, Williams TN. Sickle cell disease in Africa: a neglected cause of early childhood mortality. Am J Prev Med. 2011;41(6 Suppl 4):S398-405.

2. Jude MA, Aliyu GN, Nalado AM, et al. Stroke prevalence amongst sickle cell disease patients in Nigeria: a multi-centre study. Afr Health Sci. 2014;14(2):446-452.

3. Munube D, Katabira E, Ndeezi G, Joloba M, Lhatoo S, Sajatovic M, Tumwine JK. Prevalence of stroke in children admitted with sickle cell anaemia to Mulago Hospital. BMC Neurol. 2016;16:175.

4. Yawn BP, Buchanan GR, Afenyi-Annan AN, et al. Management of sickle cell disease: summary of the 2014 evidence-based report by expert panel members. JAMA. 2014;312(10):1033-1048.

5. Qureshi A, Kaya B, Pancham S, et al. Guidelines for the use of hydroxycarbamide in children and adults with sickle cell disease. $\mathrm{Br}$ J Haematol. 2018;181(4):460-475.

6. McGann PT, Hernandez A, Ware RE. Sickle cell anemia in subSaharan Africa: advancing the clinical paradigm through parternships and research. Blood. 2017;129(2):155-161.

7. Anyanwu JN, Williams O, Sautter CL, et al. Novel use Of Hydroxyurea in an African Region With Malaria: Protocol for a Randomized Controlled Clinical Trial. JMIR Res Protoc. 2016; 5(2):e110

8. Opoka RO, Ndugwa CM, Latham TS, et al. Novel use of hydroxyurea in an african region with malaria (NOHARM): a trial for children with sickle cell anemia. Blood. 2017;130(24):2585-2593.

9. Ware RE, Davis BR, Schultz WH, et al. Hydroxycarbamide versus chronic transfusions for maintenance of transcranial doppler flow velocities in children with sickle cell anaemia - TCD With Transfusions Changing to Hydroxyurea (TWiTCH): a multicenter, open-label, phase 3, non-inferiority trial. Lancet. 2016; 387(10019):661-670.

10. Adams R, McKie V, Nichols F, et al. The use of transcranial ultrasonography to predict stroke in sickle cell disease. $\mathrm{N}$ Engl J Med. 1992;326(9):605-610.

11. Tshilolo L, Tomlinson G, Williams TN, et al. Hydroxyurea for children with sickle cell anemia in sub-Saharan Africa. N Engl J Med. 2019;380(2):121-131

12. Noubiap JJ, Mengnjo MK, Nicastro N, Kamtchum-Tatuene J. Neurologic complications of sickle cell disease in Africa: a systematic review and meta-analysis. Neurology. 2017;89(14):1516-1524.

13. Marks LJ, Munube D, Kasirye P, et al. Stroke prevalence in children with sickle cell disease in sub-Saharan Africa: a systematic review and meta-analysis. Glob Pediatr Health. 2018;5:2333794X18774970.

14. Zimmerman SA, Schultz WH, Burgett S, Mortier NA, Ware RE. Hydroxyurea therapy lowers transcranial Doppler flow velocities in children with sickle cell anemia. Blood. 2007;110(3):1043-1047.

15. Wang WC, Ware RE, Miller ST, et al. Hydroxycarbamide in very young children with sickle-cell anaemia: a multicenter, randomised, controlled trial (BABY HUG). Lancet. 2011;377(9778):1663-1672. 